\title{
A feasibility study to determine the reliability of the Malnutrition Universal Screening Tool against anthropometric indices in determining the malnutrition risk of elderly inpatients
}

\author{
C. Hubbard \\ Nutrition Standards Project, Ashludie Hospital, Monifieth, Dundee DD5 4HQ
}

The Malnutrition Universal Screening Tool (MUST) has been introduced into NHS Tayside wards specialising in the elderly. The tool has not been validated against anthropometric indices. It is important to establish if the MUST is better at predicting nutritional risk when compared to anthropometric indices, the extent of malnutrition within this patient population and whether malnourished patients are being identified by the MUST.

Thirty-seven wards were invited to take part as they had implemented the MUST. All patients were eligible to be included unless terminally ill, unable to consent or they refused to participate. The researcher undertook anthropometric measurements of the patients: mid arm circumference (MAC) and triceps skinfold thickness (TSF). The latest MUST score was obtained from the nursing care plan. Nutritional status was determined by applying established criteria $^{(1,2)}$ and placed into one of three categories. Ethical approval was granted.

Of the nineteen wards that chose to be involved, seventy-six patients were recruited with 189 being excluded for the reasons above. Applying anthropometric criteria, $40 \%$ were either overweight or obese, $42 \%$ were of desirable weight, $17 \%$ were malnourished and $1 \%$ was unable to have nutritional status determined as height and BMI had not been recorded. Using the MUST, 67\% of patients were at low risk of malnutrition, $15 \%$ at medium risk and $17 \%$ at high risk with $1 \%$ not having the MUST completed. Comparisons between the MUST and nutritional status were made for seventy-four patients:

\begin{tabular}{|c|c|c|c|c|c|c|c|}
\hline \multirow[b]{2}{*}{ MUST } & \multicolumn{2}{|c|}{$\begin{array}{l}\text { Desirable weight } \\
\text { Overweight Obese }\end{array}$} & \multicolumn{2}{|c|}{$\begin{array}{c}\text { Mildly malnourished/BMI } \\
<20 \mathrm{~kg} / \mathrm{m}^{2} \text { with skinfolds }>10^{\text {th }} \text { centile }\end{array}$} & \multicolumn{2}{|c|}{$\begin{array}{l}\text { Moderately/severely } \\
\text { malnourished }\end{array}$} & \multirow{2}{*}{$\begin{array}{c}\text { Total } \\
n\end{array}$} \\
\hline & $n$ & $\%$ & $n$ & $\%$ & $n$ & $\%$ & \\
\hline Low & 50 & 82 & 0 & & 0 & & 50 \\
\hline Medium & 8 & 13 & 3 & 30 & 0 & & 11 \\
\hline High & 3 & 5 & 7 & 70 & 3 & 100 & 13 \\
\hline Total & 61 & 100 & 10 & 100 & 3 & 100 & 74 \\
\hline
\end{tabular}

Kappa $=0.415$ indicating fair to good agreement.

It is evident that $18 \%$ of those who were of desirable weight, overweight or obese were at medium or high risk of malnutrition as they had either reported weight loss or poor nutritional intake. All of the patients deemed malnourished by anthropometry were identified by MUST as at nutritional risk.

It appears that the MUST highlights $32 \%$ of patients as at risk of malnutrition compared to $17 \%$ using anthropometry. There appears to be a problem of overweight and obesity within this group. Future work should concentrate efforts at both under and over nutrition and continued implementation of the MUST.

1. McWhirter JP \& Pennington CR (1994) British Medical Journal 308, 945-948.

2. Elia M (editor) (2003) The 'MUST' report - Nutritional screening of adults: a multidisciplinary responsibility. Redditch: BAPEN. 